\title{
Performance Comparison of Single and Multiple Watermarking Techniques
}

\author{
Mohananthini Natarajan \\ Department of Electrical Engineering, Annamalai University, Tamilnadu, India \\ Email: mohananthini@yahoo.co.in \\ Yamuna Govindarajan \\ Department of Electrical Engineering, Annamalai University, Tamilnadu, India \\ Email: yamuna.sky@gmail.com
}

\begin{abstract}
The present work compares single and multiple watermarking by using discrete wavelet transform and different embedding methods. The different embedding methods are additive, multiplicative and hybrid watermarking with importance on its robustness versus the imperceptibility of the watermark. The objective quality metrics are demonstrated that, the additive embedding method achieves superior performance against watermark attacks on multiple watermarking technique.
\end{abstract}

Index Terms - Watermarking, additive, multiplicative, hybrid, attacks, discrete wavelet transform.

\section{INTRODUCTION}

Most of the early methods were based on single watermark embedding, but there are great limitations when single watermark embedding algorithms are tried into practical applications in few rare situation, like when multiple users share the copyright, it is need to support multiple users to embed their watermarks synchronously. This highlights the needs for multiple watermark embedding. To advocate several goals, the multiple watermarks are embedded into the image; to achieve the robustness on image processing operations is the desired one.

Embedding method is an important issue in a watermarking scheme. The embedding methods are classified into additive, multiplicative and quantization watermarking. The additive watermarking is characterized by the linear modification of the host image and the correlative processing in the detection stage. The multiplicative watermarking embeds the watermarks into image with multiplicative operation.

The third category of watermarking methods which use neither additive nor multiplicative formulas. The quantization watermarking is to perform non-linear modifications and detect the embedded message by quantizing the received samples to map them to the nearest reconstruction point. There has been many works in the literature related to watermarking techniques and some of them are discussed below.

Agreste and Puccio [1] presented the difficulties occurred during the construction and the experimentation of wavelet-based watermarking algorithm. They faced the classical watermarking problems such as computational cost, imperceptibility and robustness. Gunjal et al. [2] proposed DWT-SVD based color image watermarking technique in YUV color space using arnold transform. Their proposed scheme is non blind and strongly robust to different attacks like compression, scaling, rotation, cropping and Noise addition. Rosline Nesakumari et al. [3] proposed a novel image watermarking technique, a trusted authority to recover the ownership from any reasonably attacks. Their proposed HCPI-MBSPVD method requires less computational power, higher robustness, high payload and imperceptibility.

In [4], Safabakhsh et al. proposed a digital watermarking on still images using wavelet transform. Peter et al. has proposed single, multiple and iterative watermark embedding schemes [5]. Experimental results shows that the three watermark embedding algorithms give watermarked images with good visual quality. An integer wavelet based multiple logo-watermarking schemes for copyright protection of digital image is presented in [6]. Their method shows better robustness to attacks. Experimental study on watermark interference in multiple re-watermarking is presented in [7]. Their method shows the watermarks are all embedded into the same frequency band because it is preferable to use different embedding domains.

The collusion resistance of the fingerprinting algorithm based on additive watermarking is analyzed in [8]. Their method shows the robustness against various collusion attacks. Antonis Mairgiotis et al. [9] proposed a new family of watermark detectors for additive watermarks in digital images. Their numerical experiments demonstrate that these new detectors can lead to superior performance to several state-of-the-art detectors. Antonis Mairgiotis et al. proposed the application of a hierarchical prior for the multiplicative image watermarking problem [10]. Their investigated the detector's performance against intentional or unintentional attacks. Van Schyndel et al. [11] proposed, the embedding process is additive in the angular domain and multiplicative in the data domain. Florent Autrusseau et al. presented a hybrid watermarking technique which mixes additive and 
multiplicative watermark embedding with emphasis on its robustness versus the imperceptibility of the single watermark [12]. Their experimental results showed that using a hybrid watermarking technique significantly improves the robustness performance. Ikeda et al. proposed a hybrid scheme shows a better performance than both additive and multiplicative ones in the DWT domain [13].

Mong-Shu Lee has presented two image processing applications such as compression and watermarking, by exploiting the localization property of wavelet transform [14]. Experimental results show that their approach is quite effective in authenticating the origin of an image. Embedding binary visualized image into the original image by modifying coefficients of wavelet domain in LL bands with appropriate strength factor in order to compromise between acceptable imperceptibility level and attacks resistance are presented [15]. Their method achieves robustness level of various attacks such as image processing and rotation attacks. Tapas Bandyopadhyay et al. [16] proposed the imperceptibility quality of the images is quite good after embedding the watermark and subsequent compression. Their method of watermarking is robust against common signal processing attacks and geometric attacks.

This paper compares a multiple watermarking technique with single watermarking technique by using different embedding methods. The experimental result shows that, the PSNR and image quality are degraded with every one new watermark embedded into image and to achieve more robustness on multiple watermarking. This paper is organized as follows; the watermark embedding and extraction process are explained in section II. The experimental results and discussion are explained in section III. Conclusion of the present work is given in section IV.

\section{WATERMARK EMBEDDING AND EXTRACTION}

Embedding works are performed the multiple watermarks are embed into the original image by using the discrete wavelet transform. The watermarks used for embedding is a binary logo image, which is small compared with the size of the original image. The watermark extraction processes are the inverse process of watermark embedding. The different embedding methods are discussed below.

\section{A. Discrete wavelet transform}

The wavelet transform is based on small waves of the multi resolution analysis. Multi resolution analysis is the decomposition of an image into sub images of different size resolution levels. The proposed scheme is based on two-dimensional discrete wavelet transform, each level of produces four bands of data, one corresponding to the approximation sub-band (LL), and other three corresponding to details sub-bands such as, horizontal (HL), vertical (LH), and diagonal (HH) sub-bands. The decomposed image shows an approximation sub-band (LL) in the lowest resolution low pass band. The low pass band can further be decomposed to obtain another level of decomposition. Fig. 1 shows general two level wavelet decomposition.

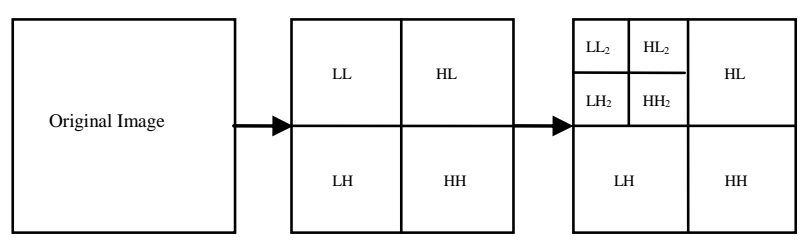

Fig. 1. General two level wavelet Decomposition

\section{B. Watermark embedding methods}

\section{Additive watermarking}

Most of the early watermarking techniques were based on an additive embedding method. In additive watermarking, the watermark is multiplied with a global embedding strength and added to the wavelet image coefficients.

The additive embedding equation is given by

$$
I_{W}(i, j)=I(i, j)+\alpha \times W(i, j)
$$

Where, IW = watermarked image,

$$
\begin{aligned}
& \mathrm{W}=\text { watermark, } \\
& \mathrm{I}=\text { cover Image and } \\
& \alpha=\text { scaling factor }
\end{aligned}
$$

The additive extraction equation is given by

$$
\left.W(i, j)=I_{W}(i, j)-I(i, j)\right) / \alpha
$$

The simplest approach to watermark embedding is the additive one which calculates the watermarked feature. In this method, the correlation based detector operates at optimum, that is, either the overall error probability or the false negative can be minimized.

\section{Multiplicative watermarking}

More current watermarking techniques apply a multiplicative embedding method. In multiplicative watermarking, the watermark embedded into the wavelet coefficients are locally modulated proportional to the strength of the coefficients. The multiplicative embedding equation is given by

$$
I_{W}(i, j)=I(i, j)(1+\alpha \times W(i, j))
$$

The multiplicative extraction equation is given by

$$
\left.W(i, j)=I_{W}(i, j)-I(i, j)\right) /(\alpha \times I(i, j))
$$




\section{Hybrid watermarking}

In hybrid watermarking, combine the additive and multiplicative watermark embedding methods. In hybrid watermarking allows us to have large watermark strength while keeping the imperceptibility is high. The hybrid embedding equation is given by

$$
\left.I_{W}(i, j)=I(i, j)+(\alpha \times I(i, j)+\beta) \times W(i, j)\right)
$$

Here $\beta$ is watermark strength to improve the correlation of watermark extraction. The hybrid extraction equation is given by

$$
\left.W(i, j)=I_{W}(i, j)-I(i, j)\right) /(\alpha \times I(i, j)+\beta)
$$

\section{Watermarking techniques}

\section{Single watermarking technique}

Embedding process: The original image is decomposed by two levels by using discrete wavelet transform. The watermark is converted into binary image and embedded in $\mathrm{LL}_{2}$ sub band by using the additive, multiplicative and hybrid embedding equations are (1), (3), and (5). The inverse wavelet transform is performed to get the watermarked image.

Extraction process: The watermarked image and the original image are decomposed by two levels by using discrete wavelet transform. The watermark can be extracted from the watermarked image in $\mathrm{LL}_{2}$ sub band by using the additive, multiplicative and hybrid extraction equations are (2), (4), and (6).

\section{Multiple watermarking technique}

Embedding process: The original image is decomposed by two levels by using discrete wavelet transform. The first watermark is converted into binary image and embedded in $\mathrm{LL}_{2}$ sub band by using the additive, multiplicative and hybrid embedding equations are (1), (3), and (5). The inverse wavelet transform is performed to get the watermarked image1. Similarly, the second watermark is embedded into the watermarked images 1 , to get the watermarked image 2 .

Extraction process: The watermarked image 2 and the watermarked image 1 are decomposed by two levels by using discrete wavelet transform. The second watermark can be extracted from the watermarked image 2 in $\mathrm{LL}_{2}$ sub band by using the additive, multiplicative and hybrid extraction equations are (2), (4), and (6). The first watermark is extracted from the watermarked images 1 and original image by repeating the above steps.

\section{EXPERIMENTAL RESULTS AND DISCUSSION}

To compare the performance of single and multiple watermarking techniques based on additive, multiplicative and hybrid embedding methods can be evaluated by peak signal to noise Ratio (PSNR) and
Normalized Correlation (NC).

Fig. 2 (a) shows $512 \times 512$ size of peppers as the original image and $48 \times 48$ size gray scale logo is used as watermark images that are shown in Fig. 2 (b) and Fig. 2 (c).

The PSNR is used to measure the quality of watermarked images, which is given by

$$
\operatorname{PSNR}(d B)=10 \log _{10} \frac{255^{2}}{M S E}
$$

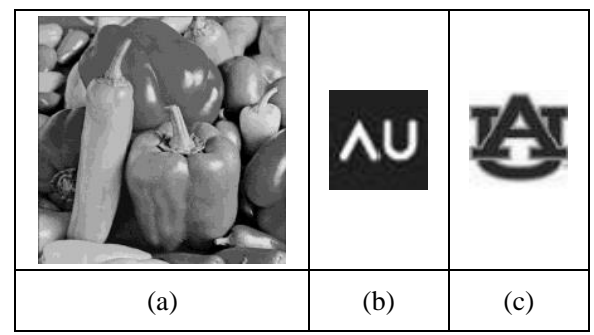

Fig. 2. (a) Peppers (b) First watermark (c) Second watermark

$\mathrm{NC}$ is used to measure the quality of watermarks after extraction, which is given by The $\mathrm{NC}$ between the embedded watermark $\mathrm{W}(\mathrm{i}, \mathrm{j})$ and the extracted watermark $W^{\prime}(i, j)$ is defined as

$$
N C=\frac{\sum_{i=1}^{H} \sum_{j=1}^{L} W(i, j) \times W^{\prime}(i, j)}{\sum_{i=1}^{H} \sum_{j=1}^{L}[W(i, j)]^{2}}
$$

To prove the robustness, the watermarked images are tested for the selected attacks such as salt \& pepper noise, Gaussian noise, median filtering, translation, cropping, rotation, JPEG compression, sharpening, smoothing, and blurring. Table 1 shows the PSNR and NC values for without attacks on single and multiple watermarking technique by using three embedding methods. Fig. 3 shows comparison of PSNR values for three embedding methods on single and multiple watermarking. The result demonstrates that the additive embedding method achieves more imperceptibility on single and multiple watermarked images, when compared with multiplicative and hybrid embedding methods.

Table 2 shows the PSNR and NC values for common image processing attacks on single watermarking technique by using three embedding methods. Fig. 4 shows the comparison of NC values for single watermarking on selected attacks. The experimental results demonstrate that the hybrid embedding method achieves more robustness on single watermarked images, when compared with additive and multiplicative embedding methods.

Table 3 shows the PSNR and NC values for common image processing attacks on multiple watermarking technique by using three embedding methods.

Fig. 5 shows the comparison of $\mathrm{NC} 2$ values for multiple watermarking on selected attacks. The 
experimental result demonstrates that the additive embedding method achieves more robustness on second watermark of multiple watermarking technique, when compared with multiplicative and hybrid embedding methods. As a result, we have to embed more than one watermark that is multiple watermarks suited for only additive embedding method achieves more visual quality and robustness.

Table 1. PSNR and NC values for watermarked images and extracted watermarks on single and multiple watermarking

\begin{tabular}{|c|c|c|c|c|c|c|}
\hline \multirow[b]{2}{*}{$\begin{array}{l}\text { Different } \\
\text { embedding } \\
\text { methods }\end{array}$} & \multicolumn{2}{|c|}{ Single watermarking } & \multicolumn{4}{|c|}{ Multiple watermarking } \\
\hline & $\begin{array}{l}\text { Watermarked image } \\
\text { PSNR }(\mathrm{dB})\end{array}$ & $\begin{array}{c}\text { Extracted } \\
\text { watermark } \\
\mathrm{NC}\end{array}$ & $\begin{array}{c}\text { Watermarked image1 } \\
\text { PSNR }(\mathrm{dB})\end{array}$ & $\begin{array}{c}\text { Watermarked image } 2 \\
\text { PSNR }(\mathrm{dB})\end{array}$ & $\begin{array}{c}\text { Extracted } \\
\text { watermark1 } \\
\text { NC1 }\end{array}$ & $\begin{array}{c}\text { Extracted } \\
\text { watermark2 } \\
\text { NC2 }\end{array}$ \\
\hline \multirow[t]{2}{*}{$\begin{array}{l}\text { Additive } \\
\text { method }\end{array}$} & & & & & & \\
\hline & 47.36 & 1 & 47.36 & 42.02 & 1 & 1 \\
\hline \multirow[t]{2}{*}{$\begin{array}{l}\text { Multiplicative } \\
\text { method }\end{array}$} & & & & & & \\
\hline & 46.02 & 1 & 46.02 & 41.40 & 1 & 1 \\
\hline \multirow[t]{2}{*}{$\begin{array}{l}\text { Hybrid } \\
\text { method }\end{array}$} & & & & & & \\
\hline & 45.81 & 1 & 45.81 & 45.81 & 1 & 1 \\
\hline
\end{tabular}

PSNR (dB)

$\square$ Additive $\square$ Multiplicative $\square$ Hybrid

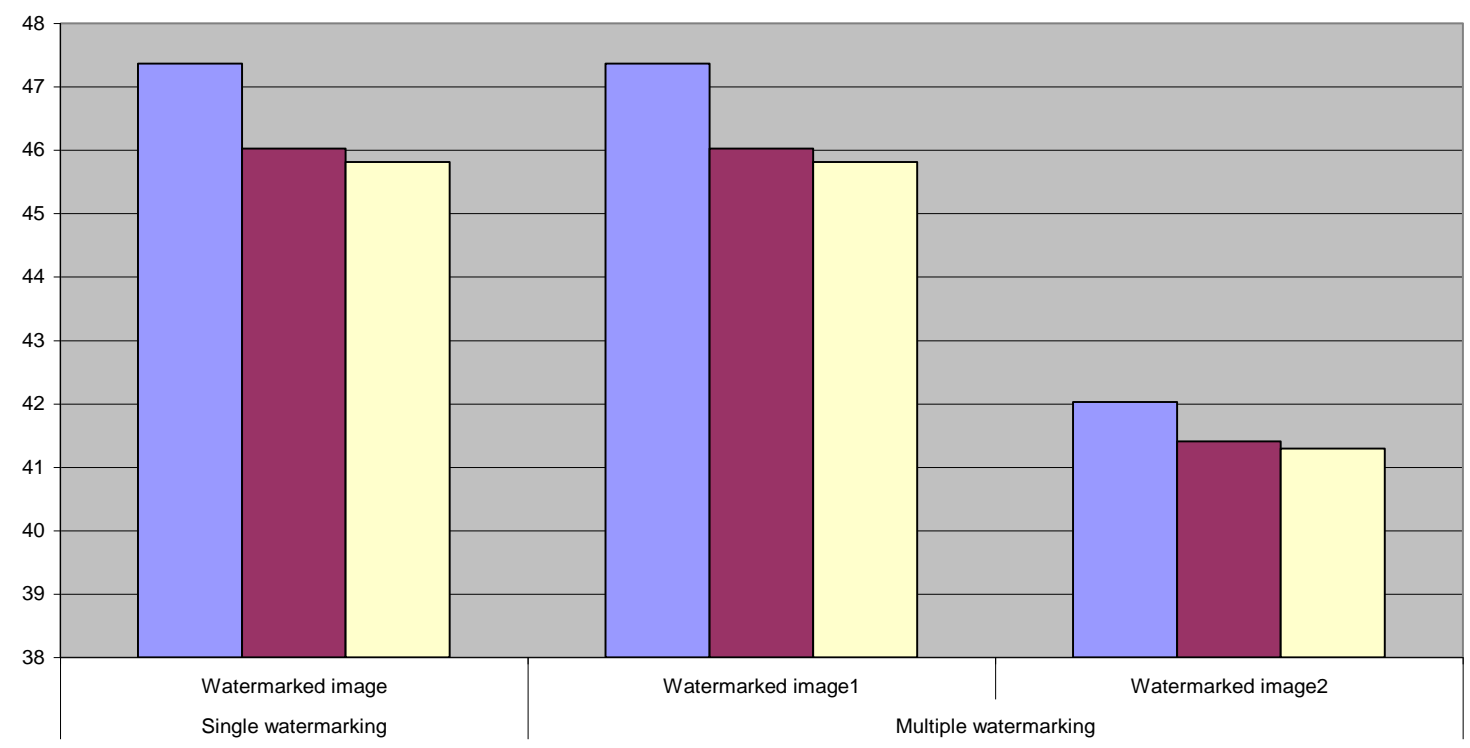

Fig. 3. Comparison of PSNR values for single and multiple watermarking 
Table 2. PSNR and NC values for common image processing attacks on single watermarking

\begin{tabular}{|c|c|c|c|c|c|c|}
\hline \multirow{2}{*}{ Attacks } & \multicolumn{2}{|c|}{$\begin{array}{c}\text { Additive } \\
\text { method }\end{array}$} & \multicolumn{2}{c|}{ Multiplicative method } & \multicolumn{2}{c|}{ Hybrid method } \\
\cline { 2 - 7 } & PSNR(dB) & NC & PSNR(dB) & NC & PSNR(dB) & NC \\
\hline $\begin{array}{c}\text { Salt \& pepper noise } \\
\text { at the density of 5\% }\end{array}$ & 17.10 & 0.738 & 17.07 & 0.706 & 17.04 & 0.754 \\
\hline $\begin{array}{c}\text { Gaussian noise } \\
\text { of variance 5\% }\end{array}$ & 14.13 & 0.634 & 14.10 & 0.619 & 14.11 & 0.657 \\
\hline $\begin{array}{c}\text { Median filtering } \\
\text { For 3 } \times 3 \text { filter size }\end{array}$ & 37.90 & 0.829 & 37.85 & 0.912 & 37.88 & 0.908 \\
\hline Translation & 21.58 & 0.384 & 21.58 & 0.392 & 21.58 & 0.392 \\
\hline Cropping & 10.84 & 0.650 & 10.85 & 0.650 & 10.85 & 0.650 \\
\hline Rotation at 60 degrees & 11.10 & 0.547 & 11.11 & 0.547 & 11.11 & 0.543 \\
\hline $\begin{array}{c}\text { JPEG compression } \\
\text { with quality of 90 }\end{array}$ & 43.13 & 1 & 42.87 & 1 & 42.82 & 1 \\
\hline Sharpening & 27.26 & 0.881 & 27.23 & 0.928 & 27.22 & 0.928 \\
\hline Smoothing & 42.85 & 0.916 & 42.63 & 0.972 & 42.58 & 0.980 \\
\hline Blurring & 32.07 & 0.66 & 32.06 & 0.730 & 32.05 & 0.734 \\
\hline
\end{tabular}

Single watermarking

口Additive $\square$ Multiplicative 口Hybrid

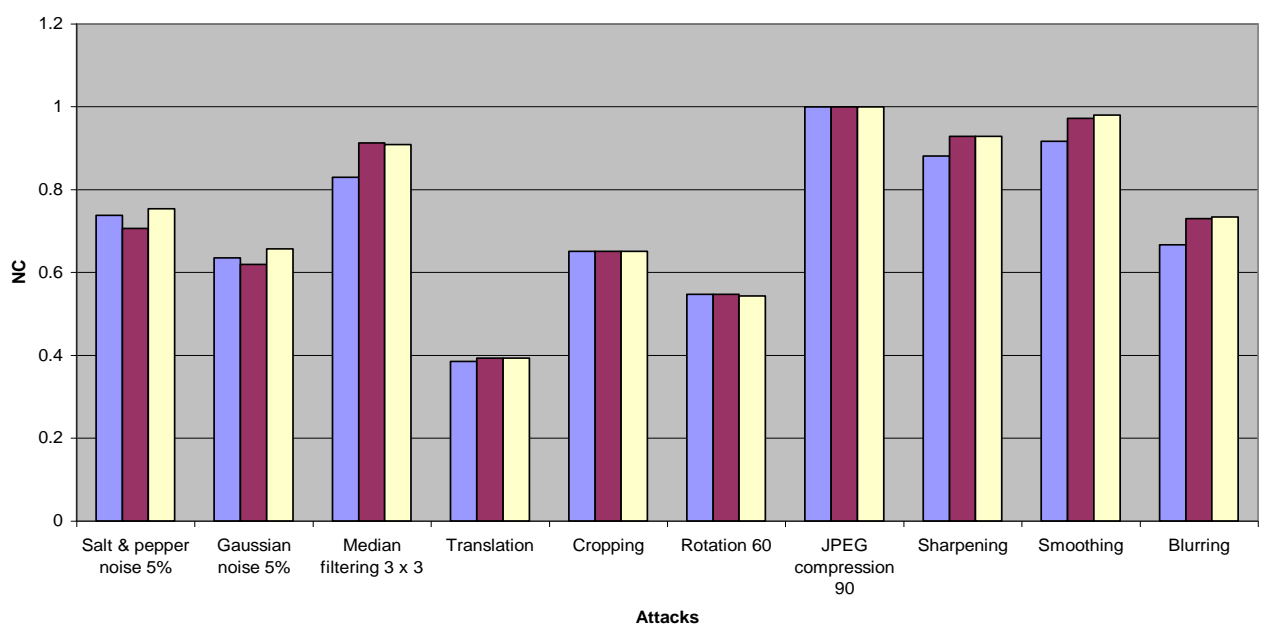

Fig. 4. Comparison of NC values for single watermarking on selected attacks

Table 3. PSNR and NC values for common image processing attacks on multiple watermarking

\begin{tabular}{|c|c|c|c|c|c|c|c|c|c|c|c|c|}
\hline \multirow{2}{*}{ Attacks } & \multicolumn{4}{|c|}{ Additive method PSNR(dB) } & \multicolumn{4}{|c|}{ Multiplicative method PSNR(dB) } & \multicolumn{4}{|c|}{ Hybrid method PSNR(dB) } \\
\hline & $\mathrm{IW}_{1}$ & $\mathrm{NC1}$ & $\mathrm{IW}_{2}$ & $\mathrm{NC} 2$ & $\mathrm{IW}_{1}$ & $\mathrm{NC} 1$ & $\mathrm{IW}_{2}$ & $\mathrm{NC} 2$ & $\mathrm{IW}_{1}$ & $\mathrm{NC} 1$ & $\mathrm{IW}_{2}$ & $\mathrm{NC2}$ \\
\hline $\begin{array}{c}\text { Salt \& pepper noise at the density } \\
\text { of } 5 \%\end{array}$ & 17.10 & 0.73 & 16.99 & 0.42 & 17.10 & 0.75 & 16.99 & 0.42 & 17.04 & 0.76 & 17.03 & 0.42 \\
\hline $\begin{array}{l}\text { Gaussian noise } \\
\text { Of variance } 5 \%\end{array}$ & 14.12 & 0.59 & 14.06 & 0.24 & 14.12 & 0.59 & 14.06 & 0.25 & 14.11 & 0.65 & 14.09 & 0.25 \\
\hline $\begin{array}{l}\text { Median filtering } \\
\text { for } 3 \times 3 \text { filter size }\end{array}$ & 37.75 & 0.81 & 37.20 & 0.95 & 37.68 & 0.92 & 37.12 & 0.96 & 38.04 & 0.93 & 37.16 & 0.97 \\
\hline Translation & 19.05 & 0.35 & 19.05 & 0.99 & 19.06 & 0.35 & 19.05 & 0.99 & 19.06 & 0.35 & 19.05 & 0.99 \\
\hline Cropping & 10.84 & 0.65 & 10.82 & 0.85 & 10.85 & 0.65 & 10.83 & 0.81 & 10.85 & 0.65 & 10.83 & 0.81 \\
\hline Rotation at 60 degrees & 11.10 & 0.54 & 11.11 & 0.90 & 11.11 & 0.54 & 11.11 & 0.86 & 11.11 & 0.54 & 11.11 & 0.86 \\
\hline $\begin{array}{c}\text { JPEG compression with quality } \\
\text { of } 90 \\
\end{array}$ & 43.13 & 1 & 40.59 & 1 & 42.87 & 1 & 40.38 & 1 & 42.82 & 1 & 40.34 & 1 \\
\hline Sharpening & 27.26 & 0.88 & 27.11 & 0.99 & 27.23 & 0.92 & 27.06 & 0.99 & 27.22 & 0.92 & 27.05 & 0.99 \\
\hline Smoothing & 42.85 & 0.91 & 40.44 & 1 & 42.63 & 0.97 & 40.26 & 1 & 42.58 & 0.98 & 40.22 & 1 \\
\hline Blurring & 32.07 & 0.66 & 31.84 & 1 & 32.06 & 0.73 & 31.83 & 1 & 32.05 & 0.73 & 31.82 & 1 \\
\hline
\end{tabular}




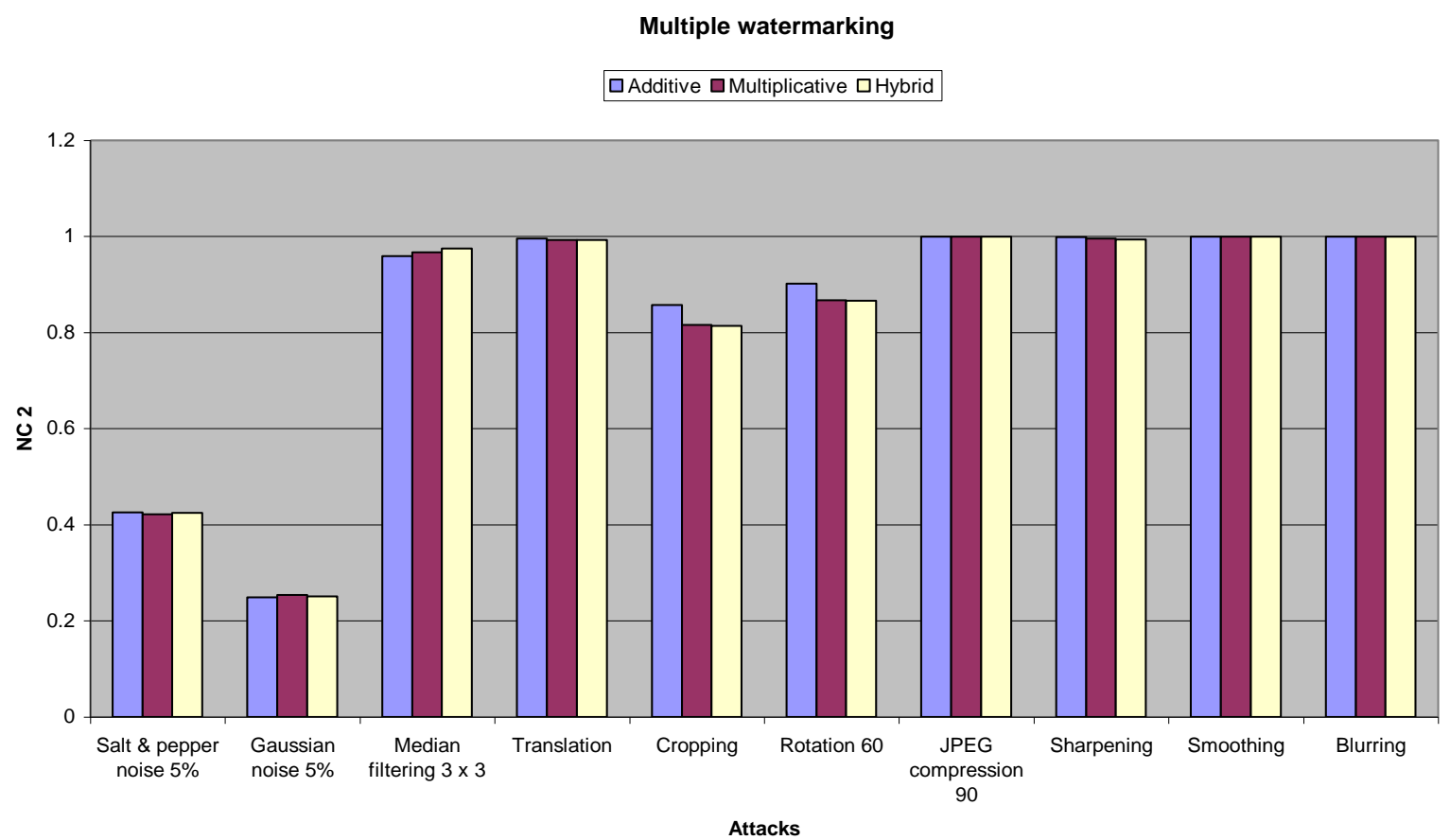

Fig. 5. Comparison of NC2 values for multiple watermarking on selected attacks

Multiple watermarking (Additive)

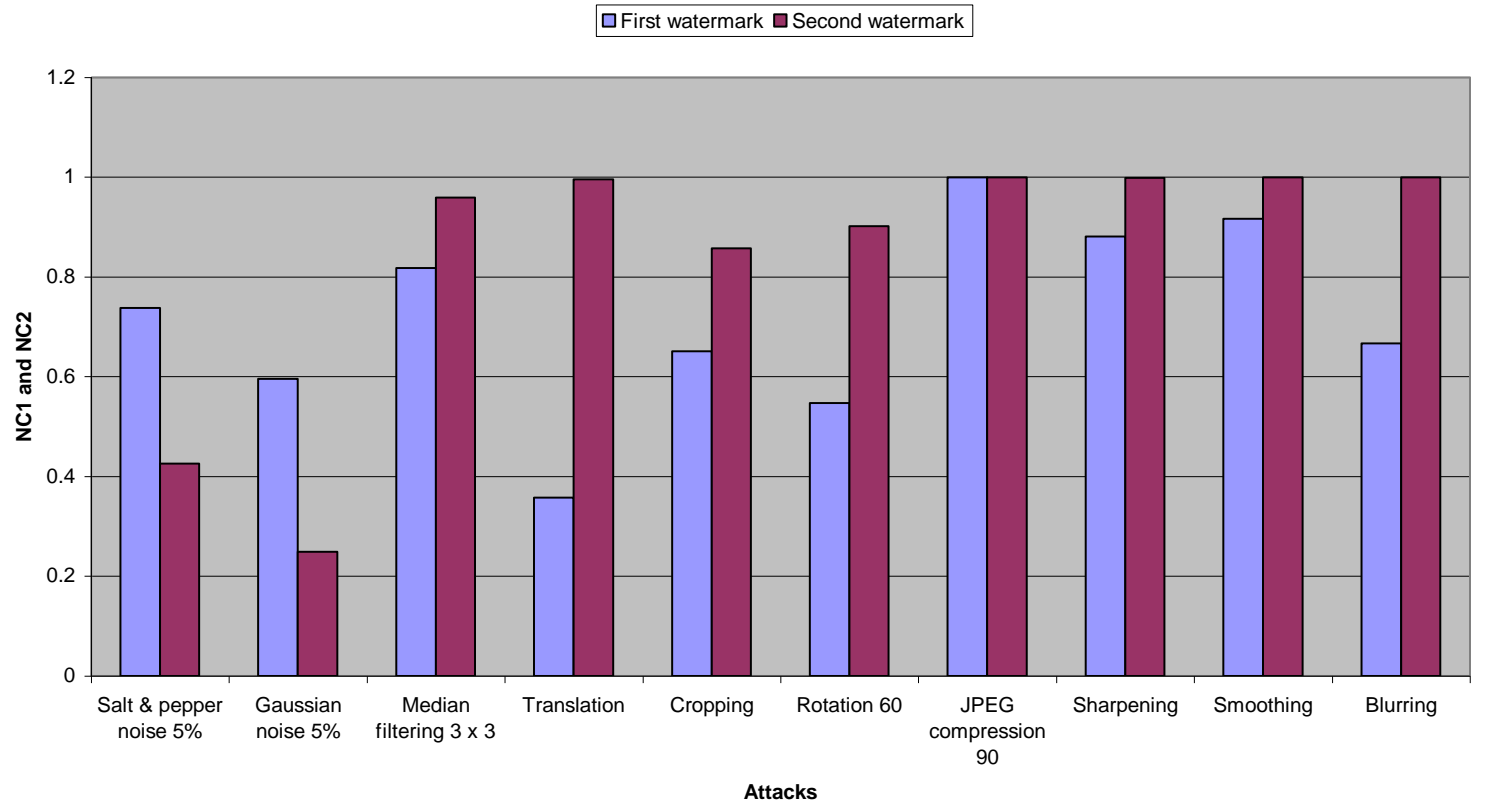

Fig. 6. Comparison of $\mathrm{NC} 1$ and $\mathrm{NC} 2$ values for multiple watermarking on selected attacks

Fig. 6 shows the comparison of $\mathrm{NC1}$ and $\mathrm{NC} 2$ values for multiple watermarking on selected attacks. In multiple watermarking technique achieves more robustness on second watermark, when compared with first watermark. Therefore multiple watermarking technique achieves more robustness, when compared with single watermarking technique. 
embedding method achieves more robustness on multiple watermarking technique. The experimental results show that the additive embedding method achieves better visual quality and robustness on multiple watermarking technique.

\section{REFERENCES}

[1] S. Agreste and L. Puccio, "Wavelet-based watermarking algorithms: theory, applications and critical aspects," International Journal of Computer Mathematics, Vol. 88, No. 9, 2011, pp. 1885-1895.

[2] Baisa L. Gunjal and Suresh N. Mali, "Strongly Robust and Highly Secured DWT-SVD Based Color Image Watermarking: Embedding Data in All Y, U, V Color Spaces," International Journal of Information Technology and Computer Science, Vol. 3, 2012, pp. 17.

[3] Rosline Nesakumari. G, Vijayakumar. V and Ramana Reddy. B. V, "Generation of an Efficient Digital Watermark Key Based on Honey Comb Polynomial Interpolation Approach", International Journal of Computer Network and Information Security, Vol. 3, 2013, pp. 47-55.

[4] R. Safabakhsh, S. Zaboli and A. Tabibiazar, "Digital watermarking on still images using Wavelet transform," International Conference on Information Technology: Coding and Computing ITCC 2004, Las Vegas.

[5] Peter H. W. Wong, Oscar C. Au and Y. M. Yeung, "A Novel Blind Multiple Watermarking Technique for Images", IEEE Transactions on Circuits and Systems for Video Technology, Vol. 13, No. 8, 2003.

[6] Yuan Yuan, Decai Huang and Duanyang Liu, "An Integer Wavelet Based Multiple Logo-watermarking Scheme", First International Multi-Symposiums on Computer and Computational Sciences, IMSCCS 2006, Hanzhou.

[7] Daniel Mark, Andreas Uhl and Hartmut Wernisch, "Experimental Study on Watermark Interference in Multiple Re-Watermarking, Security, Steganography and Watermarking of Multimedia Contents", Proceedings of the SPIE, 2007, San Jose.

[8] $\mathrm{Xi}$ Chen and Shiguo Lian, "On the CollusionResistance of the Fingerprinting Algorithm Based on Additive Watermarking," Journal of Multimedia, Vol. 6, No. 4, 2011, pp. 369-375.

[9] Antonis K. Mairgiotis, Nikolaos P. Galatsanos and Yongyi Yang, "New Additive Watermark Detectors Based on A Hierarchical Spatially Adaptive Image Model", IEEE Transactions on Information Forensics and Security, Vol. 3, No. 1, 2008, pp. 29-37.

[10] Antonis Mairgiotis, George Stylios and Constantinos Constantinopoulos, "Blind transform-based multiplicative watermarking through a hierarchical prior", Third International Conference on Circuits, Systems, Control Signals, CSCS 2012, Barcelona.

[11] R.G. Van Schyndel, A.Z. Tirkel and I. D. Svalbe, "A Multiplicative Colour Watermark", IEEE-EURASIP
Workshop on Nonlinear Signal and Image Processing, NSIP 1999, Antalya.

[12] Florent Autrusseau, Sylvain David, Vinod Pankajakshan and Patrizio Campisi, "A perceptually driven hybrid additive-multiplicative watermarking technique in the wavelet domain", SPIE Media Watermarking, Security and Forensics III SPIE, 2011, California.

[13] S Ikeda, Suita, M Yoshida and T Fujiwara, "New hybrid additive-multiplicative watermarking with better trade off between image quality and detection accuracy", International Symposium on Information Theory and its Applications, ISITA 2012, Honolulu.

[14] Mong-Shu Lee, Image compression and watermarking by wavelet localization," International Journal of Computer Mathematics, Vol. 80, No. 4, 2010, pp. 401412.

[15] Chirawat Temi, Somsak Choomchuay and Attasit Lasakul, "A Robust Image Watermarking Using Multiresolution Analysis of Wavelet", International Symposium on Communications and Information Technology, ISCIT 2005, Beijing.

[16] Tapas Bandyopadhyay, B Bandyopadhyay and B N Chatterji, "Image security through SVD based robust watermarking and compression techniques," International Journal of Emerging Trends \& Technology in Computer Science, Vol. 1, No. 3, 2012, pp. 160-165.

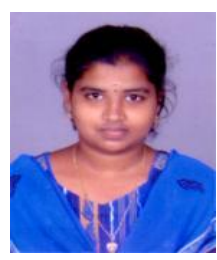

Mohananthini Natarajan received her B.E. (Electrical and Electronics) Degree from Annai Mathammal Sheela Engineering College, Namakkal, Tamil Nadu, India in 2007. She received her M.E. (Applied Electronics) Degree from Anna University, Chennai, Tamilnadu, India in 2009. Her current research areas are digital image processing and information security.

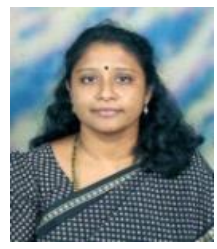

Yamuna Govindarajan received her B.E. (Electronics and Communication) Degree from the Regional Engineering College, Trichy, Tamil Nadu, and India in 1987. She received her M.E. (Power Systems) Degree from Annamalai University in the year 1991. She received her Ph.D Degree in Electrical Engineering from the Annamalai University in the year 2010. She has published many technical papers in national and international conferences and journals. Currently, she is working as Professor in the Department of Electrical and Electronics Engineering, Annamalai University, Tamil Nadu, India. Her areas of interest are digital signal processing, digital image processing and information security.

How to cite this paper: Mohananthini Natarajan, Yamuna Govindarajan,"Performance Comparison of Single and Multiple Watermarking Techniques", IJCNIS, vol.6, no.7, pp.28-34, 2014. DOI: 10.5815/ijcnis.2014.07.04 